\title{
Analytical forms of the deuteron wave function for Nijmegen group potentials and polarization characteristics of $A\left(d, d^{\prime}\right) X$ reactions
}

\author{
V. I. Zhaba \\ Uzhgorod National University, Department of Theoretical Physics, \\ 54, Voloshyna St., Uzhgorod, UA-88000, Ukraine
}

\begin{abstract}
Polarization observables of the A(d,d')X reactions have been calculated according to the received coefficients of the analytical form for deuteron wave function in coordinate space for the nucleon-nucleon Nijmegen group potentials (NijmI, NijmII, Nijm93). The obtained values of tensor $A_{y y}$ and vector $A_{y}$ analyzing powers have been compared with the published experimental data at $t$ - scaling for the inelastic scattering of deuterons on hydrogen, carbon and beryllium. Theoretical values of tensor-tensor $K_{y y}$ and vector-vector $K_{y}$ polarization transfers have been also evaluated in the plane-wave impulse approximation.
\end{abstract}

Keywords: analytical forms, deuteron, impulse approximation, analyzing powers, polarization transfers.

PACS 03.65.Nk, 13.40.Gp, 13.88.+e, 21.45.Bc

\section{Introduction}

Deuteron - is the simplest core, which consists of the two elementary particles - a proton and a neutron. The simplicity and clarity of the deuteron structure serves as a convenient laboratory for simulation and analyzing nucleon-nucleon forces. Despite a detailed theoretical and experimental study, the deuteron is of considerable interest today due to certain theoretical inconsistencies. In particular, according to the review [1] in a number of papers, the deuteron wave function (DWF) in coordinate representation has knots near the origin of coordinates. The existence of such knots of the ground and the only state of the deuteron attests to inconsistencies and inaccuracies in the implementation of numerical algorithms in solving such problems, or to the features of potential deuteron models 2 .

In the review [1, the static parameters of the deuteron obtained from DWF for various potential models have been systematized, and a review of the analytical forms of DWF in coordinate representation has been carried out. What is more, both analytical forms and parameterizations of DWF have been presented, which are necessary for further calculations of the processes characteristics with the participation of deuteron. In addition, it has been noted that in such a convenient form, DWFs are needed when applied to calculate the polarization characteristics of the deuteron, as well as to evaluate the theoretical values of spin observables in dp-scattering. After all, the DWF can also be used to calculate the parameters and characteristics of the reactions of (d,d') type.

In this paper, analytical forms of DWF have been used for theoretical calculations of a set of polarization observables in $\mathrm{A}\left(\mathrm{d}, \mathrm{d}^{\prime}\right) \mathrm{X}$ reactions. Realistic phenomenological Nijmegen group potentials (NijmI, NijmII and Nijm93) [3, 4] have been used for numerical calculations. 


\section{DWF analytical forms}

Among the large list of analytical forms of DWF in the coordinate representation, parametrization of DWF for the Parisian potential [5] is worth highlighting

$$
\left\{\begin{array}{l}
u(r)=\sum_{j=1}^{N} C_{j} \exp \left(-m_{j} r\right) \\
w(r)=\sum_{j=1}^{N} D_{j} \exp \left(-m_{j} r\right)\left[1+\frac{3}{m_{j} r}+\frac{3}{\left(m_{j} r\right)^{2}}\right]
\end{array}\right.
$$

where $m_{j}=\beta+(j-1) m_{0} ; \beta=\sqrt{M E_{d}}, m_{0}=0.9 \mathrm{fm}^{-1} ; M$ - nucleon mass; $E_{d}$ - binding energy of the deuteron. Extreme conditions at $r \rightarrow 0$ :

$$
u(r) \rightarrow r ; \quad w(r) \rightarrow r^{3} .
$$

The asymptotics of the deuteron wave function (1) for $r \rightarrow \infty$

$$
\begin{aligned}
& u(r) \sim A_{S} \exp (-\beta r), \\
& w(r) \sim A_{D} \exp (-\beta r)\left[1+\frac{3}{\beta r}+\frac{3}{(\beta r)^{2}}\right],
\end{aligned}
$$

where $A_{S}$ and $A_{D}$ are the asymptotics of $S$ - and $D$ - state normalizations.

The coefficients of the analytical form (3) for $N>10$ are determined by the formulas [

$$
\left\{\begin{array}{l}
C_{n}=-\sum_{j=1}^{n-1} C_{j} \\
D_{n-2}=\frac{m_{n-2}^{2}}{\left(m_{n}^{2}-m_{n-2}^{2}\right)\left(m_{n-1}^{2}-m_{n-2}^{2}\right)}\left[-m_{n-1}^{2} m_{n}^{2} \sum_{j=1}^{n-3} \frac{D_{j}}{m_{j}^{2}}+\left(m_{n-1}^{2}+m_{n}^{2}\right) \sum_{j=1}^{n-3} D_{j}-\sum_{j=1}^{n-3} D_{j} m_{j}^{2}\right]
\end{array}\right.
$$

or

$$
\sum_{j=1}^{N_{b}} C_{j}=0 ; \quad \sum_{j=1}^{N_{b}} D_{j}=\sum_{j=1}^{N_{b}} D_{j} m_{j}^{2}=\sum_{j=1}^{N_{b}} \frac{D_{j}}{m_{j}^{2}}=0 .
$$

The search for the coefficients of the analytical form (1) was done for the Paris [5] and the Bonn (OBEPC 6] and charge-dependent Bonn (CD-Bonn) [7) potentials and the fss2 model (with the Coulomb exchange kernel [8], and calculated according to three different schemes (isospin basis and particle basis with or without the Coulomb force and fss2 baryon-baryon interaction 9]), with $N=13,11$ and 11 respectively. What is more, formula (1) was applied to MT model [10], where $N_{S}=16 ; N_{D}=12$.

The new analytical DWFs were also proposed in coordinate representation in the 2000s. These include, for instance, Dubovichenko's [1] and BerezhnoyKorda-Gakh's [12, parameterizations, as well as the analytic form in such a simple form 13 . 


$$
\left\{\begin{array}{l}
u(r)=r \sum_{i=1}^{N} A_{i} \exp \left(-a_{i} r^{2}\right), \\
w(r)=r \sum_{i=1}^{N} B_{i} \exp \left(-b_{i} r^{2}\right) .
\end{array}\right.
$$

The DWF (1) was used to approximate the numerical arrays of radial wave functions obtained for Nijmegen group potentials (NijmI, NijmII, Nijm93 and Reid93). The behavior of the $\chi^{2}$ value depending on the number of terms of the $N$ expansion has been studied. In addition to the DWF (2), the analytical form for Nijmegen group potentials (NijmI, NijmII and Nijm93) was suggested in paper [14]

$$
\left\{\begin{array}{l}
u(r)=r^{3 / 2} \sum_{i=1}^{N} A_{i} \exp \left(-a_{i} r^{3}\right), \\
w(r)=r \sum_{i=1}^{N} B_{i} \exp \left(-b_{i} r^{3}\right) .
\end{array}\right.
$$

Formula (3) was used in paper 2] for approximation of DWFs for the Reid93 and Argonne v18 potentials. The obtained wave functions for these potentials do not contain any superfluous knots. Theoretical evaluation of the polarization characteristics of the deuteron using DWF (3) is appropriate.

DWF parameters in the dressed dibaryon model (DDM) 15] were obtained for the analytical form [16]

$$
\left\{\begin{array}{l}
u(r)=r \sum_{i=1}^{N} A_{i} \exp \left(-a_{i} r^{2}\right), \\
w(r)=r^{3} \sum_{i=1}^{N} B_{i} \exp \left(-b_{i} r^{2}\right) .
\end{array}\right.
$$

\section{Polarization observables of the $A\left(d^{\prime}, d^{\prime}\right) X$ reactions}

Experimental determination of the values of polarization characteristics for the deuteron fragmentation reaction of $\mathrm{A}(\mathrm{d}, \mathrm{p}) \mathrm{X}$ and for $(\mathrm{d}, \mathrm{d}$ ') reactions of inelastic scattering of the deuteron on nuclei [17] remain one of the main tools for studying the deuteron structure. In addition, the reactions of inelastic scattering of deuterons are also used to study the properties and the formation of baryonic resonances.

The singlet breakup contribution to the amplitude and others elastic scattering observables for ${ }^{58} \mathrm{Ni}(\mathrm{d}, \mathrm{d}){ }^{58} \mathrm{Ni}$ reaction at deuteron energy $400 \mathrm{MeV}$ is studied in paper [18. The inclusion of the singlet channel coupling produce a large effect on the calculated angular distributions for reaction tensor analyzing power $A_{y y}$ but insignificant effect on the cross section and vector analyzing power $A_{y}$. It takes place through dynamically induced $T_{L}$ tensor interaction. Tensor and vector analyzing powers for deuterons occurring from $\mathrm{d}+{ }^{58} \mathrm{Ni}$ inelastic scattering as a function of outgoing deuteron energy are provided in [19].

Within the framework of the plane-wave impulse approximation model (PWIA) [17] previously obtained experimental data [20] of tensor and vector analyzing 
powers have been analyzed in the reaction of inelastic scattering of deuterons on carbon at an initial momentum of deuteron of $9 \mathrm{GeV} / \mathrm{c}$ and a detection angle of secondary deuterons of $85 \mathrm{mrad}$ in the region of resonance excitation with a mass of $2190 \mathrm{MeV} / \mathrm{c}^{2}$.

The results of experimental studies of the tensor $A_{y y}$ and vector $A_{y}$ analyzing powers for inelastic scattering of deuterons with a momentum of $4.5 \mathrm{GeV} / \mathrm{c}$ on beryllium at an angle of $80 \mathrm{mrad}$ around the excitation of baryonic resonances are provided in [21]. The $A_{y y}$ values are in good agreement with the experimental data at a zero angle and show an approximate $t$ - scaling up to $\sim-0.9(\mathrm{GeV} / \mathrm{c})^{2}$. Paper [22] provides the experimental data of analyzing powers in inelastic scattering of deuterons with a momentum of $5.0 \mathrm{GeV} / \mathrm{c}$ on beryllium at an angle of $178 \mathrm{mrad}$ in the vicinity of excitation of baryonic resonances with a mass up to $\sim 1.8 \mathrm{GeV} / \mathrm{c}^{2}$. Moreover, the values of $A_{y y}$ correlate well with the previous results at 4.5 and $5.5 \mathrm{GeV} / \mathrm{c}$. The results of these experiments are compared with the predictions of models with multiple-scattering, PWIA and $\omega$-meson exchange models.

The angular dependence of analyzing powers in the inelastic scattering of deuterons with a momentum of $9.0 \mathrm{GeV} / \mathrm{c}$ on hydrogen and carbon has been measured in paper [23]. The measurement range corresponds to a baryonic resonance excitation with a mass of $2.2-2.6 \mathrm{GeV} / \mathrm{c}^{2}$. These data show approximate $t$ - scaling up to $-1.5(\mathrm{GeV} / \mathrm{c})^{2}$. The large figures for $A_{y}$ value suggested a significant role of the spin-dependent part of the elementary amplitude of the $\mathrm{NN} \rightarrow \mathrm{NN}^{*}$ reaction.

The values of the tensor $A_{y y}$ and vector $A_{y}$ analyzing powers can be received experimentally from the numbers of deuterons $n^{+}, n^{-}, n^{0}$, registered for different modes of beam polarization $p_{z}, p_{z z}$ and normalized to the beam intensity, taking into account the dead-time effect of the installation [22]:

$$
\begin{aligned}
& A_{y y}=2 \frac{p_{z}^{-}\left(n^{+} / n^{0}-1\right)-p_{z}^{+}\left(n^{-} / n^{0}-1\right)}{p_{z}^{-} p_{z z}^{+}-p_{z}^{+} p_{z z}^{-}} . \\
& A_{y}=-\frac{2}{3} \frac{p_{z z}^{-}\left(n^{+} / n^{0}-1\right)-p_{z z}^{+}\left(n^{-} / n^{0}-1\right)}{p_{z}^{-} p_{z z}^{+}-p_{z}^{+} p_{z z}^{-}} .
\end{aligned}
$$

Tensor and vector analyzing powers in PWIA are theoretically calculated according to the following formulas [17:

$$
\begin{gathered}
A_{y y}=\frac{T_{00}^{2}-T_{11}^{2}+4 P^{2} T_{10}^{2}}{T_{00}^{2}+2 T_{11}^{2}+4 P^{2} T_{10}^{2}} ; \\
A_{y}=2 \sqrt{2} P \frac{\left(T_{11}+T_{00}\right) T_{10}}{T_{00}^{2}+2 T_{11}^{2}+4 P^{2} T_{10}^{2}} ;
\end{gathered}
$$

where $T_{i j}(p / 2)$ are the amplitudes determined by the radial wave functions of the deuteron in coordinate representation $u(r)$ and $w(r)$ :

$$
T_{00}=S_{0}(p / 2)+\sqrt{2} S_{2}(p / 2),
$$




$$
\begin{gathered}
T_{11}=S_{0}(p / 2)-\frac{1}{\sqrt{2}} S_{2}(p / 2), \\
T_{10}=\frac{i}{\sqrt{2}} \int_{0}^{\infty}\left(u^{2}-\frac{w^{2}}{2}\right) j_{0} d r+\frac{i}{2} \int_{0}^{\infty} w\left(u+\frac{w}{\sqrt{2}}\right) j_{2} d r .
\end{gathered}
$$

Here $S_{0}$ and $S_{2}$ - spherical (charge) and quadrupole deuteron form factors

$$
\begin{gathered}
S_{0}(p / 2)=S_{0}^{1]}+S_{0}^{2} ; \\
S_{2}(p / 2)=2 S_{2}^{11}-\frac{1}{\sqrt{2}} S_{2}^{2 \sqrt{2}},
\end{gathered}
$$

which are expressed through elementary spherical $S_{0}^{(i)}$ and quadrupole $S_{2}^{(i)}$ form factors [17, 24] in the form

$$
\begin{aligned}
& S_{0}^{\sqrt{1]}}=\int_{0}^{\infty} u^{2} j_{0} d r ; \quad S_{0}^{\sqrt{2}}=\int_{0}^{\infty} w^{2} j_{0} d r ; \\
& S_{2}^{\sqrt[1]{1}}=\int_{0}^{\infty} u w j_{2} d r ; \quad S_{2}^{[\sqrt[2]{2}}=\int_{0}^{\infty} w^{2} j_{2} d r .
\end{aligned}
$$

Here $j_{0}, j_{2}$ - spherical Bessel functions of zero and second order from the pr/2 argument; $p$ - momentum. Besides, formulas (11) and (12) have been written down in paper [24] with $p r$ argument.

According to [17, a parameter $P=a \cdot p$ is introduced in the formulas (7) and (8), which characterizes the ratio of the spin-dependent and spin-independent parts of amplitudes for the $\mathrm{NN} \rightarrow \mathrm{NN}^{*}(2190)$ process:

$$
P=\frac{f^{s f}(p)}{f^{n f}(p)} .
$$

Here amplitudes $f^{s f}(p)$ and $f^{n f}(p)$ can be parametrized in such form [17]

$$
\begin{aligned}
& f^{s f}(p)=i \sqrt{\frac{A_{s}}{\pi}} \exp \left(-\frac{B_{s} p^{2}}{2}\right) ; \\
& f^{n f}(p)=i \sqrt{\frac{A_{n}}{\pi}} \exp \left(-\frac{B_{n} p^{2}}{2}\right) ;
\end{aligned}
$$

where $A_{s}, B_{s}, A_{n}, B_{n}$ are constants. Values of constants $A_{n}$ and $B_{n}$ can be determined from experimental data by excitation of a resonance $\mathrm{N}^{*}(2190)$ in ppinteractions. If parameters $B_{s}$ and $B_{n}$ for exponents coincide, then parameter $P$ will be linear function. 
Within the $\omega$-meson exchange model [26, 27, the tensor analyzing power in deuteron inelastic scattering is defined as [21, 25.

$$
A_{y y}=\frac{V_{1}^{2}+\left(2 V_{0} V_{2}+V_{2}^{2}\right) \rho}{4 V_{1}^{2}+\left(3 V_{0}^{2}+V_{2}^{2}+2 V_{0} V_{2}\right) \rho},
$$

where $\rho=\sigma_{L} / \sigma_{T}$ - ratio of the cross sections of absorption of virtual isoscalar photons with longitudinal and transversal polarizations by nucleons [27; the structure functions $V_{0}, V_{1}$ and $V_{2}$ is expressed in terms of the electric $G_{C}$, magnetic $G_{M}$ and quadrupole $G_{Q}$ deuteron form factors.

For $\mathrm{N}^{*}$ resonance excitation, the ratio $\rho$ can be written in the form [27, 21 ]

$$
\rho_{N *}=\frac{\left|A_{l}^{p}+A_{l}^{n}\right|^{2}}{\left|A_{1 / 2}^{p}+A_{1 / 2}^{n}\right|^{2}+\left|A_{3 / 2}^{p}+A_{3 / 2}^{n}\right|^{2}},
$$

where $A_{l}^{N}$ - longitudinal form factor of the $\mathrm{N}^{*}$ excitation on a proton $(N=p)$ or a neutron $(N=n) ; A_{1 / 2}^{N} \quad A_{3 / 2}^{N}$ - two transversal form factors, corresponding to total $\gamma^{*}+\mathrm{N}$ helicity equal to $1 / 2$ and $3 / 2$. It has been specified in paper [25] that the tensor analyzing power $A_{y y}$ in $\omega$-meson exchange model in the $t$ channel can be represented as the product of two parts that are determined by the electromagnetic deuteron properties and by the form factors for the $\mathrm{N} \rightarrow \mathrm{N}^{*}$ transition.

Using the DWF (3) for Nijmegen group potentials (the coefficients of expansions are taken from paper [14), the theoretical values of the tensor $A_{y y}$ and vector $A_{y}$ analyzing powers in PWIA have been calculated by formulas (7) and (8), respectively. Moreover, for the parameter $P$, the argument $a=0.4$ for $A_{y y}$ and $a=0.4 \div 1.1$ for $A_{y}$ has been selected. The results of numerical calculations are shown in Figures 1-4, where $t$ - scaling according to the elementary record is used according to the formula $t=-(0.197326 p)^{2}$ given units for momentum $p$ in $\left[\mathrm{fm}^{-1}\right]$ and $t$ - scaling in $\left[(\mathrm{GeV} / \mathrm{c})^{2}\right]$. The theoretical estimates of the $A_{y y}$ and $A_{y}$ values are compared with the experimental data for the reaction of (d,d') type on light nuclei: on hydrogen [23, carbon [23, 28, 29], beryllium [21, 22, 25]. Based on the data [29, the tensor analyzing power is determined by the formula: $A_{y y}=-T_{20} / \sqrt{2}$. In contrast to the calculated value of the tensor analyzing power $A_{y y}$, its vector component $A_{y}$ strongly depends on the parameter $P$ and better coincides with the experiment at $a=0.4$.

The tensor analyzing power $A_{y y}$ according to formula $(7)$ is weakly dependent on the parameter $P$. If the spin-dependent part of the amplitude in the formula for the parameter (13) is equal to zero, then formula (7) will be written as 17 ]

$$
A_{y y}=\frac{1}{2} \frac{S_{2}^{2}(p / 2)+2 \sqrt{2} S_{0}(p / 2) S_{2}(p / 2)}{S_{0}^{2}(p / 2)+S_{2}^{2}(p / 2)} .
$$

That is, tensor analyzing power $A_{y y}$ is determined only by the spherical and quadrupole deuteron form factors. Calculations by formulas (7) and (18) practically coincide. 
Papers [17, 23] provide the results of calculations of the analyzing powers obtained by DWF for the Paris and three (A, B, C) versions of the Bonn potentials. There, for $a=0.4$, the value $A_{y y}$ intersects zero in the region $|t|=1.0$ $1.2(\mathrm{GeV} / \mathrm{c})^{2}$, and the vector capacity $A_{y}$ calculated for the Paris potential better correlates with the experimental data than for the Bonn potential $(a=0.3-$ $0.4)$, and to $|t|=1.0(\mathrm{GeV} / \mathrm{c})^{2}$ is determined by the spin-dependent part of the amplitude of the elementary process $\mathrm{NN} \rightarrow \mathrm{NN}^{*}(2190)$.

It should be noted that the results of calculations of the analyzing powers of $A_{y y}$ and $A_{y}$ as in papers [17, 23] for the Paris and Bonn potentials, and in this paper for Nijmegen group potentials, strongly differ from the experimental data within $|t|=0-1.0(\mathrm{GeV} / \mathrm{c})^{2}$. In addition, there is a certain scatter of data of experimental points for close measurements. Of course, it would be interesting to obtain the analyzing powers of $\mathrm{A}\left(\mathrm{d}, \mathrm{d}^{\prime}\right) \mathrm{X}$ reaction on the mentioned targets with other values of the initial momentum of deuteron and the detection angles of secondary deuterons.

It has been specified in paper [17 that the deviation of the $A_{y y}$ value from the predictions of PWIA can be connected with the contribution of double rescatterings [30] (thus the rescatterings and quark exchange considerably influence the polarization observables of the reaction of inclusive ${ }^{12} \mathrm{C}(\mathrm{d}, \mathrm{p})$ breakup for kinematical region of high values of momentum in the deuteron), or with the presence of nonnucleonic degrees of freedom in the deuteron 31] (when the influence of baryon resonances as admixture to the deuteron on the momentum dependences of observables in high-power backward elastic dp- scattering is considered).

In paper 23] the obtained experimental data of analyzing powers have been analyzed and it has been stated that data for values $A_{y y}$ at $|\mathrm{t}| \leq 0.8(\mathrm{GeV} / \mathrm{c})^{2}$ differ from PWIA [17] calculations for standard DWFs and from the data received in dp- and ed- elastic scatterings, that is, such behavior and difference indicates to the sensitivity of $A_{y y}$ to baryonic resonance excitation through double-collision interactions.

The values of the tensor-tensor and vector-vector polarization transfers can be calculated as well, respectively [17:

$$
\begin{aligned}
& K_{y y}=\frac{5 T_{11}^{2}+T_{00}^{2}-8 P^{2} T_{10}^{2}}{T_{00}^{2}+2 T_{11}^{2}+4 P^{2} T_{10}^{2}} ; \\
& K_{y}=2 \frac{T_{00} T_{11}+2 P^{2} T_{10}^{2}}{T_{00}^{2}+2 T_{11}^{2}+4 P^{2} T_{10}^{2}} .
\end{aligned}
$$

Unfortunately, we failed to compare these theoretical estimates of $K_{y y}$ and $K_{y}$ at $a=0.4$ for Nijmegen group potentials (Figures 5 and 6 ) with the experimental data, since the latter were not found in the scientific literature. Therefore, the experiments on obtaining these polarization observables remain relevant.

Calculations of the analyzing powers and the polarization transfers for $\mathrm{Ni}$ jmegen group potentials (NijmI, NijmII and Nijm93) are compared with the 
theoretical estimates for the other three models - DDM [15, fss2 8 and OBEPC [6.

In paper [26] have been calculated the vector (tensor) transfer polarization coefficients $k_{a}^{a^{\prime}}\left(k_{a a}^{a^{\prime} a^{\prime}}\right)$ (with $a=x, y$ or $z$ ) from initial to final deuterons for $d+p \rightarrow d+X$ process in $\sigma$ - and $\omega$ - exchange models. The largest sensitivity of values $k_{y}^{y^{\prime}}$ to $\rho$ is in the region $p>3 \mathrm{fm}^{-1}$ and the position of zero is strongly $\rho$ dependent.

\section{Conclusions}

The polarization observables in the reactions of $\mathrm{A}\left(\mathrm{d}, \mathrm{d}^{\prime}\right) \mathrm{X}$ type have been calculated based on the previously obtained coefficients of the analytical form of the deuteron wave function (3) in coordinate representation for phenomenological realistic nucleon-nucleon Nijmegen group potentials (NijmI, NijmII and Nijm93). Within the framework of the plane-wave impulse approximation model [17. the theoretical values of the tensor $A_{y y}$ and vector $A_{y}$ analyzing powers have been calculated. They are compared with the experimental data of the reaction of deuterons inelastic scattering on hydrogen, carbon and beryllium. What is more, the theoretical values of the tensor-tensor $K_{y y}$ and vector-vector $K_{y}$ polarization transfers have been estimated, which are sensitive to the amplitude of $\mathrm{NN} \rightarrow \mathrm{NN}^{*}(2190)$ process up to $|t|=1.5(\mathrm{GeV} / \mathrm{c})^{2}$.

In practice, it is sometimes more convenient to use the partial cross-sections $\sigma_{i}$ or the spin-flip cross-sections $\sigma_{i}^{(s f)}$ [17, 32, which are characterized by a spin flip and are determined by the very polarization observables $A_{y y}, K_{y y}, K_{y}$ obtained in this paper:

$$
\begin{gathered}
\left\{\begin{array}{l}
\sigma_{0}=\frac{1}{6}\left(2+3 K_{y}+K_{y y}\right) ; \\
\sigma_{1}=\frac{1}{9}\left(4-\left(A_{y y}+P_{y y}\right)-2 K_{y y}\right) ; \\
\sigma_{2}=\frac{1}{18}\left(4+2\left(A_{y y}+P_{y y}\right)-9 K_{y}+K_{y y}\right) ;
\end{array}\right. \\
\left\{\begin{array}{l}
\sigma_{0}^{(s f)}=\frac{1}{3}\left(-1-2 A_{y y}+6 K_{y}\right) ; \\
\sigma_{1}^{(s f)}=\frac{2}{3}\left(2+A_{y y}-3 K_{y}\right) ; \\
\sigma_{2}^{(s f)}=0 .
\end{array}\right.
\end{gathered}
$$

Finally these polarization observables (21) can be applied to determination of the spin structure of $\overrightarrow{1}+A \rightarrow \overrightarrow{1}+B$ process and cross section 33 .

$$
I(\theta, \varphi)=I_{0}(\theta)\left(1+\frac{3}{2} \sum_{j} p_{j} A_{j}(\theta)+\frac{1}{3} \sum_{j, k} p_{j k} A_{j k}(\theta)\right),
$$

where $p_{l^{\prime}}$ - the outgoing polarization components:

$$
p_{l^{\prime}} I(\theta, \varphi)=I_{0}(\theta)\left(P_{l^{\prime}}(\theta)+\frac{3}{2} \sum_{j} p_{j} K_{j}^{l^{\prime}}(\theta)+\frac{1}{3} \sum_{j, k} p_{j k} K_{j k}^{l^{\prime}}(\theta)\right)
$$


$A_{j}(\theta), A_{j k}(\theta)$ - the analyzing powers; $P_{l^{\prime}}(\theta)$ - outgoing polarization (unpolarized incident beam); $K_{j}^{l^{\prime}}(\theta), K_{j k}^{l^{\prime}}(\theta)$ - polarization transfer coefficients (tensortensor $K_{y y}$ and vector-vector $K_{y}$ polarization transfers).

The results of numerical calculations of the partial cross-sections $\sigma_{i}$ and the spin-flip cross-sections $\sigma_{i}^{(s f)}$ at $a=0.4$ are shown in Figure 7 .

DWFs for Nijmegen group potentials can be applied for calculations of tensor and vector analyzing powers and compared with their experimental data for the deuteron fragmentation reaction with a momentum of $9 \mathrm{GeV} / \mathrm{c}$ on the nuclei of hydrogen and carbon given high proton transverse momentums [34]. Calculations need to be conducted within light-front dynamics with the use of different DWFs (including relativistic DWF).

Moreover, it is of interest to conduct the comparative analysis between highprecision experimental results [35] for vector $A_{y}$ and tensor $A_{y y}, A_{x x}, A_{x z}$ analyzing powers of $d d \rightarrow{ }^{3} \mathrm{H} p$ reaction with deuterons energy of $200 \mathrm{MeV}$ in a full angular range in the center-of-mass system and theoretical calculations, made within the multiple-scattering model with the use of standard waves functions for the three-nucleon bound state and the deuteron.

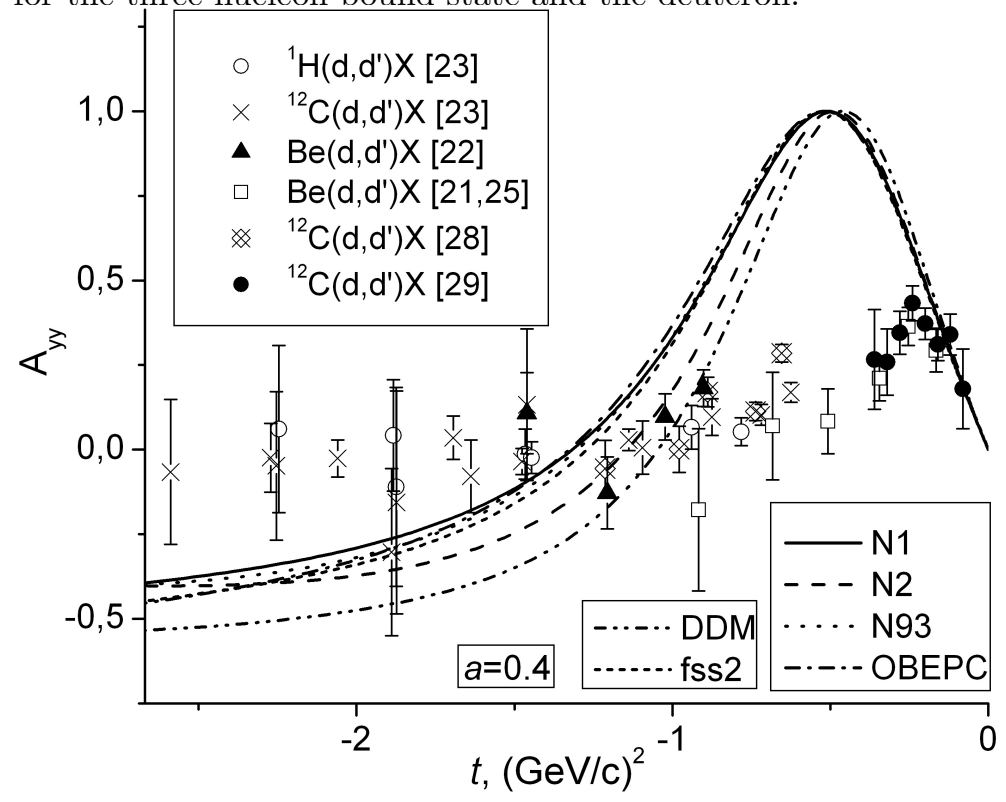




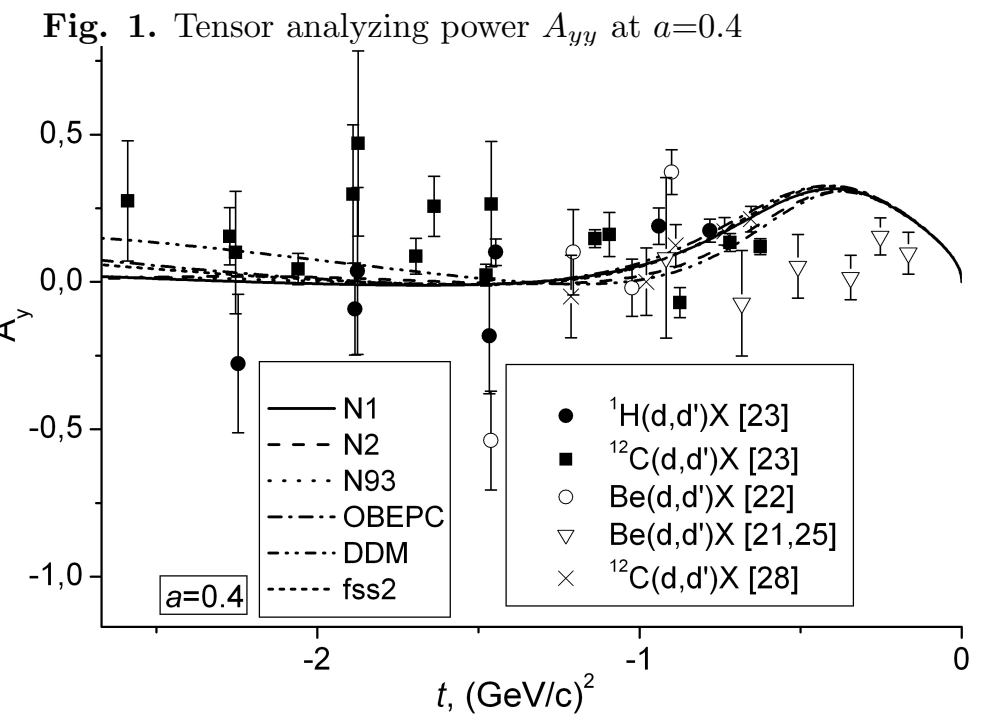

Fig. 2. Vector analyzing power $A_{y}$ at $a=0.4$

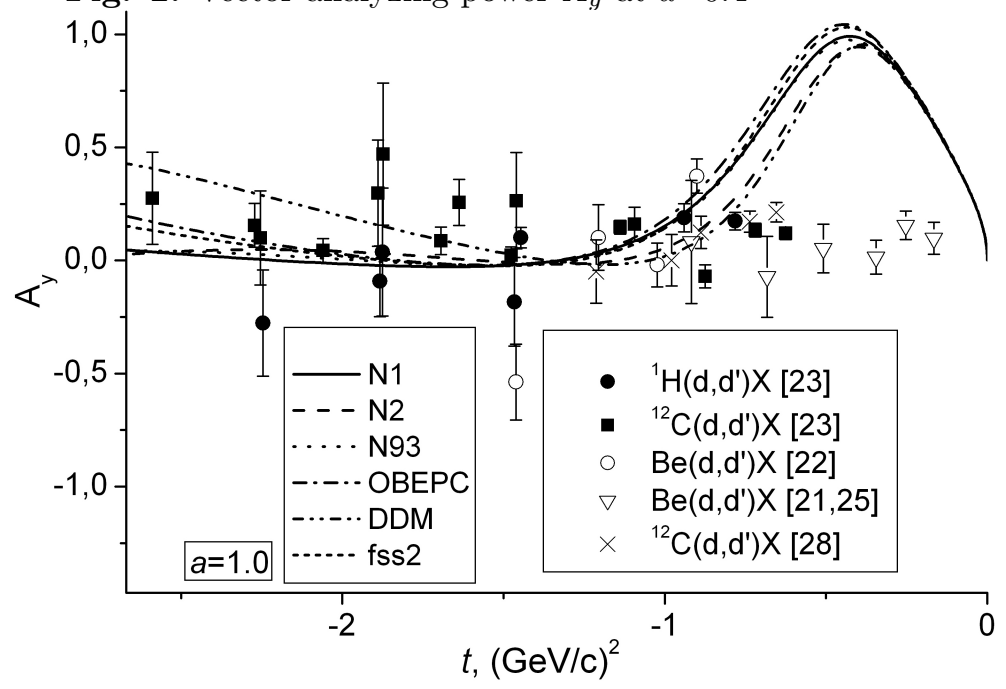




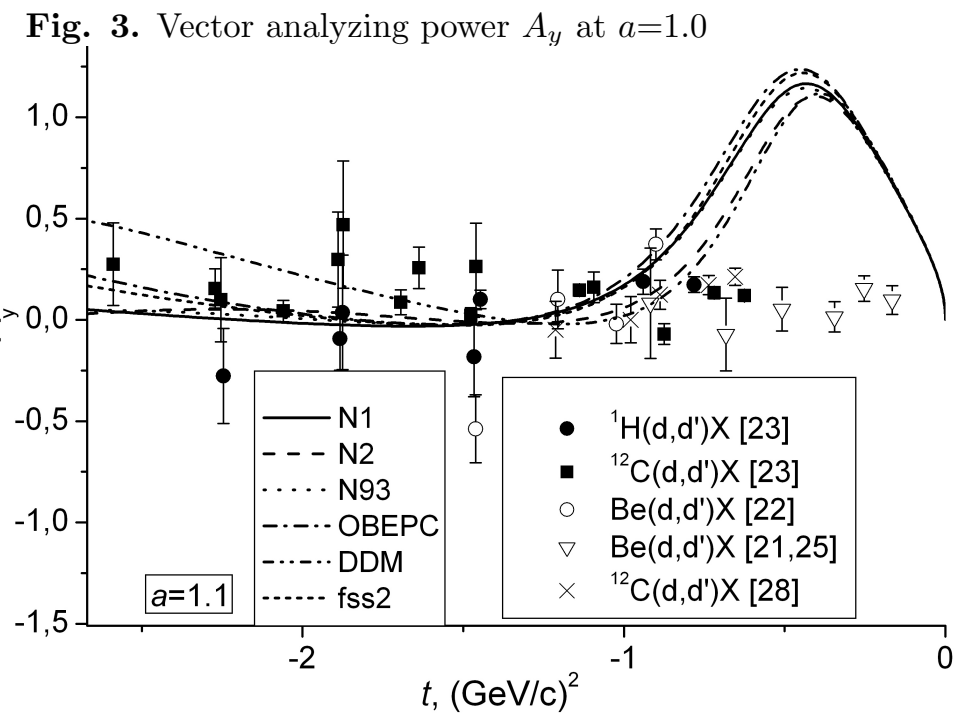

Fig. 4. Vector analyzing power $A_{y}$ at $a=1.1$

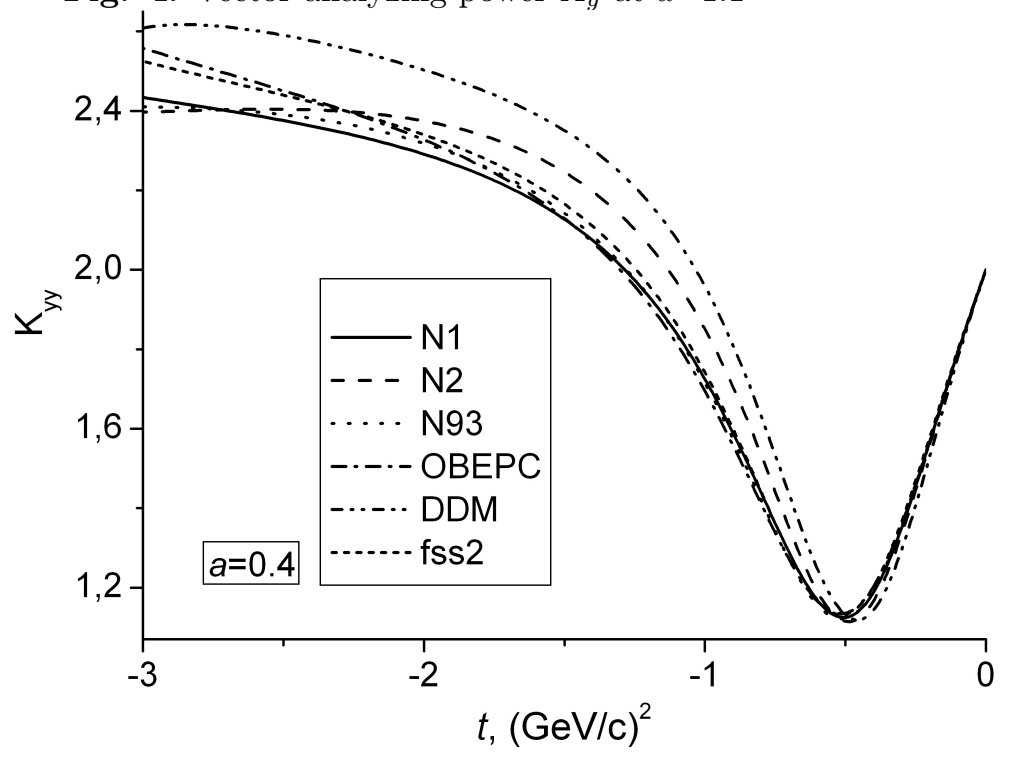




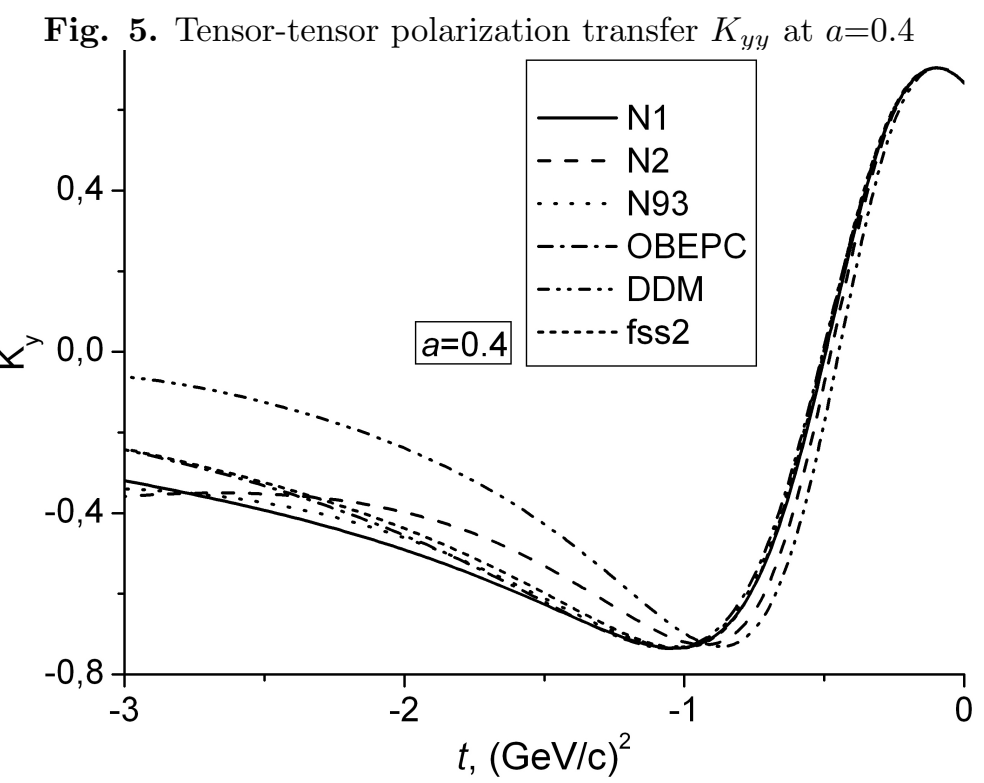

Fig. 6. Vector-vector polarization transfer $K_{y}$ at $a=0.4$

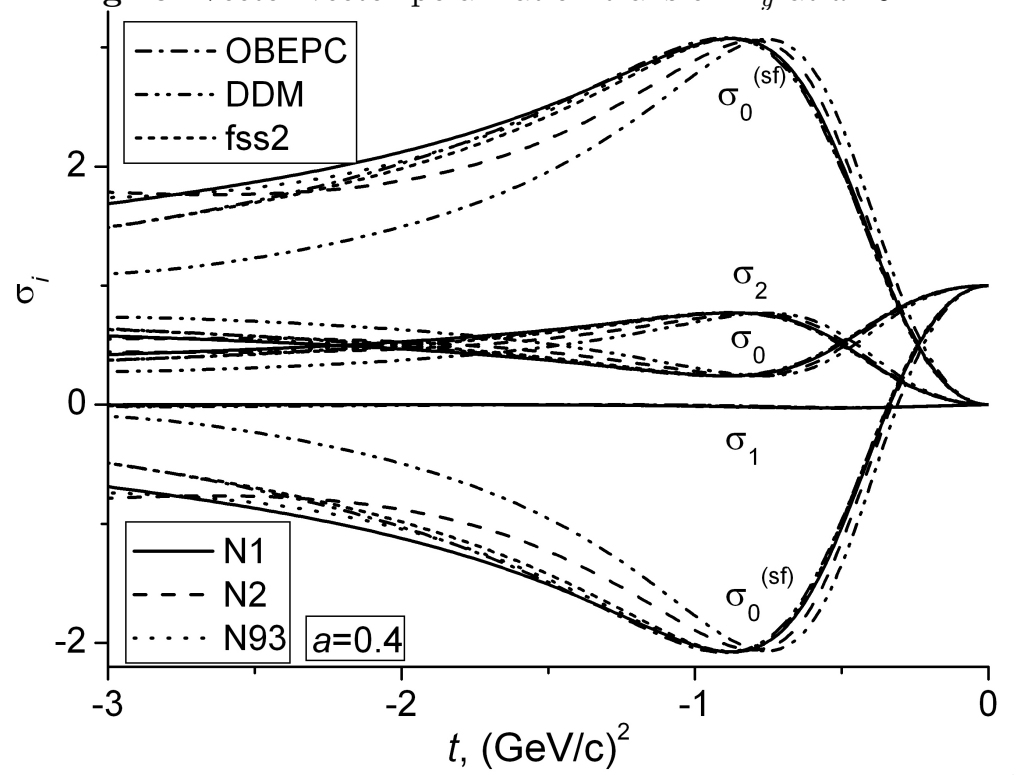

Fig. 7. Partial cross-sections $\sigma_{i}$ and spin-flip cross-sections $\sigma_{i}^{(s f)}$ at $a=0.4$

\section{References}

[1] V. I. Zhaba. Deuteron: properties and analytical forms of wave function in coordinate space // arXiv:nucl-th/1706.08306 
[2] V. I. Zhaba, Mod. Phys. Lett. A 31, 1650139 (2016).

[3] V. G. J. Stoks et al., Phys. Rev. C 49, 2950 (1994).

[4] J. J. de Swart et al., Few-Body Syst. Suppl. 8, 438 (1995).

[5] M. Lacombe et al., Phys. Lett. B 101, 139 (1981).

[6] R. Machleidt, Adv. Nucl. Phys. 19, 189 (1989).

[7] R. Machleidt, Phys. Rev. C 63, 024001 (2001).

[8] Y. Fujiwara et al., Phys. Rev. C 65, 014002 (2001).

[9] K. Fukukawa et al., Phys. Rev. C 92, 065802 (2015).

[10] A. F. Krutov and V. E. Troitsky, Phys. Rev. C 76, 017001 (2007).

[11] S. B. Dubovichenko, Phys. Atom. Nucl. 63, 734 (2000).

[12] Yu. A. Berezhnoy et al., Intern. Jour. Mod. Phys. E 14, 1073 (2005).

[13] V. I. Zhaba, J. Phys. Stud. 20, 3101 (2016).

[14] V. I. Zhaba, Nucl. Phys. Atom. Energy 17, 22 (2016).

[15] M. N. Platonova and V. I. Kukulin, Phys. Atom. Nucl. 73, 86 (2010).

[16] V. M. Krasnopol'sky et al., Phys. Lett. B 165, 7 (1985).

[17] V. P. Ladygin and N. B. Ladygina, Phys. Atom. Nucl. 65, 182 (2002).

[18] J. S. Al-Khalili et al., Phys. Rev. C 41, 806 (1990).

[19] E. J. Stephenson et al., Annual Scientific and Technical Report, p. 95-97 (1981).

[20] L. S. Azhgirey et al., Phys. Atom. Nucl. 62, 1673 (1999).

[21] V. P. Ladygin et al., Eur. Phys. J. A 8, 409 (2000).

[22] L. S. Azhgirey et al., Phys. Atom. Nucl. 68, 991 (2005).

[23] V. P. Ladygin et al., Phys. Atom. Nucl. 69, 852 (2006).

[24] M. N. Platonova and V. I. Kukulin, Phys. Rev. C 81, 014004 (2010).

[25] L. S. Azhgirey et al., Phys. Atom. Nucl. 64, 1961 (2001).

[26] M. P. Rekalo and E. Tomasi-Gustafsson, Phys. Rev. C 54, 3125 (1996).

[27] E. Tomasi-Gustafsson et al., Phys. Rev. C 59, 1526 (1999).

[28] S. V. Afanasiev et al., JINR Rapid Commun. 2[88]-98, 5 (1998). 
[29] L. S. Azhgirey et al., JINR Rapid Commun. 2[88]-98, 17 (1998).

[30] A. P. Kobushkin, Phys. Lett. B 421, 53 (1998).

[31] L. S. Azhgirey and N. P. Yudin, Phys. Atom. Nucl. 63, 2184 (2000).

[32] T. Suzuki, Nucl. Phys. A 577, 167 (1994).

[33] G. G. Ohlsen, Rep. Prog. Phys. 35, 717 (1972).

[34] L. S. Azhgirey et al., Phys. Atom. Nucl. 71, 264 (2008).

[35] A. K. Kurilkin et al., Phys. Atom. Nucl. 78, 918 (2015). 